\title{
Dinâmica populacional da mosca-dos-chifres (Haematobia irritans) em bovinos da raça Guzerá e mestiço em Selvíria, MS
}

\section{Population dynamics of the horn fly (Haematobia irritans) on Guzera cattle breed and crossbred in Selvíria, MS}

\author{
Fabiana Alves de Almeida ${ }^{1}$; Fernanda Carvalho Basso²; \\ Maria Conceição Zocoller Seno ${ }^{3 *}$; Walter Veriano Valério Filho ${ }^{4}$
}

\section{Resumo}

Objetivando conhecer a dinâmica populacional da mosca-dos-chifres em bovinos no município de Selvíria, MS, foi conduzido um experimento no período de março de 2004 a junho de 2005 na Fazenda de Ensino, Pesquisa e Extensão (FEPE), Unesp - Campus de Ilha Solteira, localizada no município de Selvíria, MS. Foram utilizadas 15 vacas da raça Guzerá e 15 mestiças (Guzerá x Holandesa), de 3 a 4 anos, naturalmente infestadas. Durante o período experimental estes animais não receberam tratamento inseticida e a cada 14 dias foi realizada a contagem visual de moscas sobre a região cervico-dorsolombar. Os resultados obtidos permitiram verificar que a mosca-dos-chifres apresentou dois picos de infestação durante o ano, um no mês de abril e o outro no mês de outubro. Nos meses de maior infestação, o número médio de moscas não ultrapassou a 104. Na comparação entre as raças, os meses em que houve diferença significativa foram abril, maio, agosto e setembro de 2004, e fevereiro, março e abril de 2005, com a raça mestiça sempre apresentando maior infestação. Na região estudada Haematobia irritans estava presente durante todo o ano.

Palavras-chave: Dinâmica populacional, mosca-dos-chifres, bovinos

\begin{abstract}
Aiming to know the population dynamics of horn fly on cattle in the municipality of Selvíria, MS, Brazil, a study was conducted from March 2004 to June 2005 in the Education, Research and Extension Farm, from Unesp - Campus de Ilha Solteira, located in the municipality of Selvíria, MS. It was used 15 cows of the Guzera breed and 15 crossbred (Guzera X Holstein-Friesian), respectively 3 and 4 years old, naturally infested. During the experimental period these animals did not receive any insecticide treatment. Visual fly counting by on back region of the animals was carried out at 14 day interval. The horn fly showed two peaks of infestation during the year, one in April and another in October. In the months of highest infestation, the average number of flies did not exceed a 104. The months in which was significant difference between crossbred and Guzera breed was in April, May, August and September 2004 and February, March and April 2005, always with crossbred with higher infestation. In the region studied Haematobia irritans was present throughout the year.
\end{abstract}

Key words: Population dynamics, horn fly, cattle

1 Mestre em Biologia Geral e Aplicada, Instituto de Biociências de Botucatu (IBB), Universidade Estadual Paulista (UNESP). E-mail: faalmeida@ibb.unesp.br

2 Mestranda em Zootecnia, Faculdade de Ciências Agrárias e Veterinárias de Jaboticabal, Universidade Estadual Paulista (UNESP).

3 Professora Adjunta, Departamento de Biologia e Zootecnia, Faculdade de Engenharia de Ilha Solteira (FEIS), Universidade Estadual Paulista (UNESP). E-mail: zocoller@bio.feis.unesp.br

${ }^{4}$ Professor Doutor, Departamento de Matemática, Faculdade de Engenharia de Ilha Solteira (FEIS), Universidade Estadual Paulista (UNESP).

* Autor para correspondência 


\section{Introdução}

Haematobia irritans, conhecida popularmente como mosca-dos-chifres, foi identificada primeiramente no Brasil no Estado de Roraima possivelmente vindo da Guiana por volta de 1977, com seu primeiro registro feito por Valério e Guimarães (1983). A sua dispersão pelo país, segundo Barros (2004) foi facilitada pelo transporte em rotas de comercialização de gado. Em 1990, notou-se a sua presença nos Estados de São Paulo e Mato Grosso do Sul e em 1991 a H. irritans chegou ao Estado do Paraná (FARIA, 1998). Hoje a mosca-dos-chifres encontra-se distribuída por todo o Brasil.

Este díptero da família Muscidae é um ectoparasita hematófago que mede de 2 a $3 \mathrm{~mm}$, machos e fêmeas se alimentam de sangue e têm preferência por sangue bovino, mas podem parasitar eqüinos e ovinos. As fêmeas deixam o animal para depositar seus ovos na massa fecal fresca, iniciando o seu ciclo de vida. A eclosão dos ovos ocorre em 24 horas, as larvas se transformam em pupas em torno de 4 dias, permanecendo no solo de 5 a 6 dias transformando-se em adultos e migrando aos hospedeiros, onde permanecerão de 6 à 8 semanas (BORDIN,1992). As picadas são dolorosas, irritam e estressam os animais, podem transmitir patógenos como carbunculose, anaplasmose e leucose (FARIA, 1998; GOMES; KOLLER; SILVA, 1998; GARCIA et al., 2001).

Os fatores abióticos, tais como temperatura e precipitação pluvial afetam a população de moscas (CORDOVÉS, 1998; GARCIA et al., 2001). Temperaturas baixas e precipitações muito baixas ou altas reduzem a intensidade do parasitismo (CORDOVÉS, 1998; GARCIA et al., 2001; BIANCHIN; ALVES, 2002).

A desproporcional distribuição da mosca-doschifres em hospedeiros individualmente tem sido atribuída às características morfofisiológicas tais como, cor da pele (FRANKS; BURNS; ENGLAND, 1964), temperatura da pele (MORGAN, 1964), cor do pelo (PRUETT et al., 2003), densidade do pelo, e secreções sebáceas (STEELMAN et al., 1997). $H$. irritans ainda tem preferência por bovinos machos adultos (FARIA, 1998) e animais de sangue europeu (FARIA, 1998; CASTRO et al., 2008) ou mestiço (FARIA, 1998).

Por seus hábitos hematófagos, estes ectoparasitos causam prejuízos aos bovinos com infestações que variam de 50 a 300 indivíduos por animal. Um animal com média de 500 moscas/dia pode sofrer uma perda anual de aproximadamente 2,6 litros de sangue, redução no ganho de peso e na produção de leite (SAUERESSIG, 1992; FARIA, 1998; GARCIA et al., 2001). Grisi et al.(2002) estimaram que as perdas causadas pela mosca-dos-chifres no Brasil era da ordem de US\$150 milhões anuais e Bianchin, Koller e Alves (2004) verificaram perdas de $10 \%$ do peso vivo nos animais da raça Nelore infestados, o que significaria segundo Bianchin, Koller e Detmann (2006), uma perda de US\$ 865 milhões. Além dessas perdas, a mosca também é responsável por uma diminuição no valor do couro (SAUERESSIG, 1992; GUGLIELMONE et al., 1999).

Devido aos prejuízos causados pela mosca, nos últimos anos tem se intensificado o controle com ouso de inseticidas químicos, principalmente piretróides, mas este tem ocorrido sem critério quanto à época e freqüência de tratamento (MARTINS et al., 2002). Segundo Martins, Porciúncula e Vieira (2002) o conhecimento da dinâmica populacional da moscados-chifres é essencial para a implementação de programas de controle que possam minimizar os prejuízos causados por este inseto.

Objetivando conhecer a dinâmica populacional da mosca-dos-chifres no município de Selvíria, MS, foi realizado o presente trabalho.

\section{Material e métodos}

O experimento foi realizado no período de março de 2004 a junho de 2005, na Fazenda de 
Ensino, Pesquisa e Extensão (FEPE), Universidade Estadual Paulista (Unesp) - Campus de Ilha Solteira, localizada no município de Selviria, MS. Para isso, foram utilizadas 15 vacas da raça Guzerá e 15 vacas mestiças (Zebu X Europeu), de três a quatro anos, naturalmente infestadas, que permaneceram em piquetes com Brachiaria decumbens.

Durante o período experimental os animais não receberam tratamento inseticida e permaneceram distantes cerca de $2 \mathrm{~km}$ do rebanho da fazenda, para que não houvesse qualquer interferência no experimento. A cada 14 dias os animais eram trazidos ao tronco, passavam no brete onde eram contidos para a realização da contagem visual de moscas sobre a região cervico-dorso-lombar (ALMEIDA et al., 2005), que consistia em contar as moscas presentes na região dorsal, desde a nuca até as pontas da anca, de cada animal.

Os dados de precipitação foram coletados na estação localizada na própria fazenda, e os dados de temperatura máxima, mínima e umidade relativa foram coletados na fazenda de produção vegetal da Universidade, distante $5 \mathrm{~km}$ da propriedade onde foi realizado o estudo.

Para a comparação entre as raças foi utilizado o modelo estatístico parcelas subdivididas (medidas repetidas no tempo), avaliando raça, mês e interação raça x mês. A análise de variância foi realizada pelo software Statistical Analysis System (SAS) através do procedimento General Linear Models (GLM). Foi realizada também uma análise descritiva comparando o número médio de moscas nos animais com os dados climáticos.

\section{Resultados e discussão}

De acordo com os dados obtidos verificou-se que a mosca-dos-chifres está presente nos animais durante o ano todo apresentando dois picos de infestação, em abril e outubro, próximo ao final e no início do período chuvoso, respectivamente, coincidindo com início das estações de outono e primavera. Resultados semelhantes foram encontrados por Lima, Perri e Prado (2003), na cidade de Araçatuba, interior de São Paulo, por Barros (2001), na região do Pantanal, MS, e por Bianchin, Koller e Detmann (2006), em Campo Grande, MS. Nota-se também que nos meses em que ocorreram os picos de infestação, abril/ 04, outubro/ 04 e abril/ 05, com exceção do volume de chuva ocorrido no mês de outubro, as condições ambientais pouco variaram. Em abril de 2004 ocorreu o primeiro pico, com média de 104 (DP \pm 79 ) moscas por animal, sendo a precipitação pluvial de $53 \mathrm{~mm}$, a umidade relativa do ar de $74 \%$ (Figura 1), a temperatura máxima de $32{ }^{\circ} \mathrm{C}$ e a mínima de $21^{\circ} \mathrm{C}$ (Figura 2 ).

De maio a agosto houve um acentuado declínio no número médio de moscas, coincidindo com o período seco, com a temperatura e umidade relativa também em declínio. Em agosto ocorreu a infestação mínima de 34 (DP \pm 21 ) moscas por animal, quando não houve precipitação, temperatura máxima $31^{\circ} \mathrm{C}$, temperatura mínima $15^{\circ} \mathrm{C}$ e umidade relativa de 56\%. Barros (2001), Bianchin e Alves (2002) encontraram resultados semelhantes no período seco do ano com baixos níveis de ocorrência da mosca-dos-chifres. Martins, Porciúncula e Vieira (2002) no Rio Grande do Sul, observaram que nos meses de junho a agosto as infestações foram nulas ou próximas de zero. Este mesmo resultado foi encontrado por Castro et al. (2008) no nordeste do Uruguai. Isto se deve ao fato de que nestas duas regiões a temperatura é muito baixa nestes meses, o que desfavorece o desenvolvimento da mosca, com as pupas entrando em diapausa (BIANCHIN; KOLLER; DETMANN, 2006). 


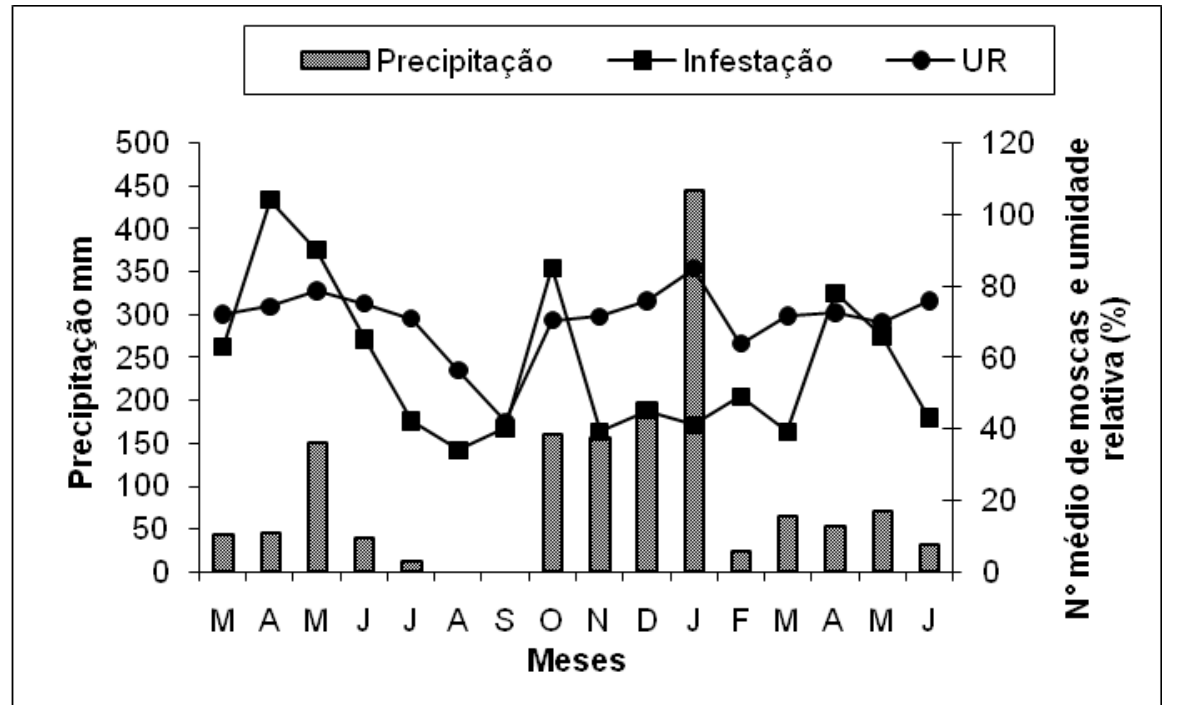

Figura 1. Precipitação pluvial, umidade relativa e número médio de mosca-dos-chifres em bovinos da raça Guzerá e Mestiço no período de março de 2004 a junho de 2005, no município de Selvíria, MS.

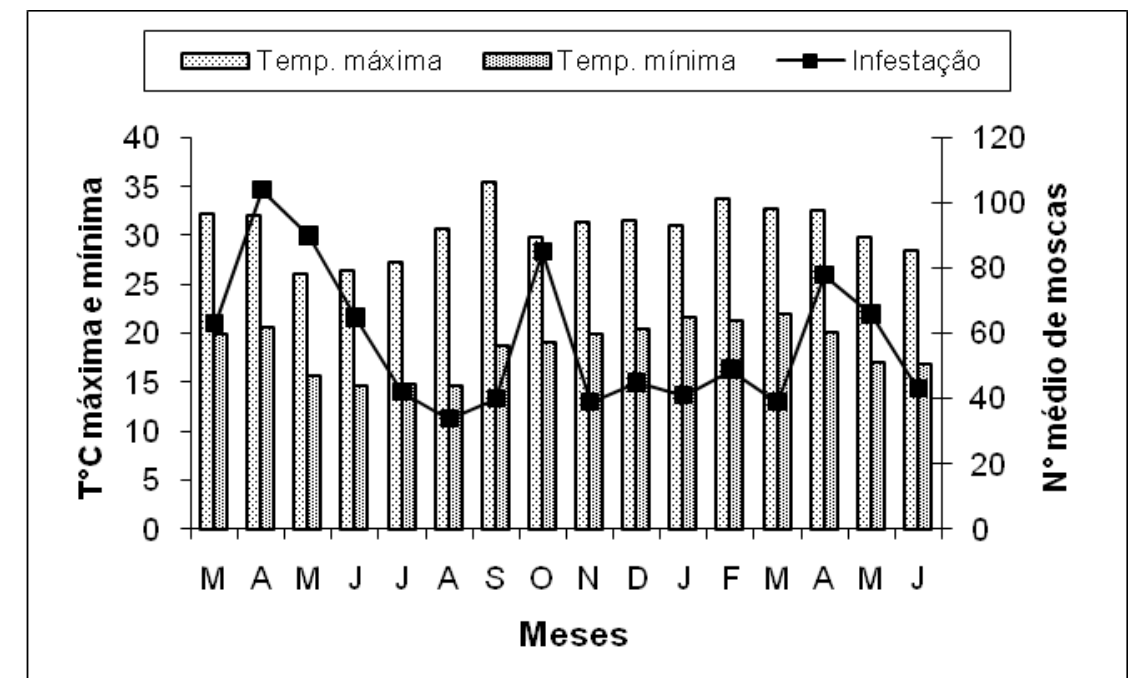

Figura 2. Temperatura máxima e mínima e número médio de mosca-dos-chifres em bovinos da raça Guzerá e Mestiço no período de março de 2004 a junho de 2005, no município de Selvíria, MS.

No mês de outubro um segundo pico de infestação foi observado, coincidindo com o início do período chuvoso, quando a média de moscas foi de 85 (DP \pm 40$)$ por animal, a precipitação de 161 $\mathrm{mm}$, temperatura máxima e mínima de $30^{\circ} \mathrm{C} \mathrm{e} 19^{\circ} \mathrm{C}$, respectivamente e umidade relativa de $70 \%$. Em abril de 2005 verificou-se novamente um pico de infestação coincidindo com o observado no ano de 2004, porém o número médio de moscas foi menor 78 (DP \pm 35 ) por animal, $54 \mathrm{~mm}$ de precipitação, $33^{\circ} \mathrm{C}$ e $20^{\circ} \mathrm{C}$ a temperatura máxima e mínima, respectivamente e $72 \%$ de umidade relativa. Nos dois últimos meses do experimento, maio e junho de 2005, notou-se um declínio no número médio de moscas, indicando uma mesma tendência ocorrida no ano anterior (Figura 1 e 2).

O resultado da análise estatística apresentou que a interação raça $x$ mês foi significativa $(P<0,01)$, e desta forma optou-se pela comparação das raças dentro de cada mês. Pelo Teste F, os meses em 
que houve diferenças significativas foram abril, maio, agosto e setembro de 2004, e fevereiro, março e abril de 2005, com a raça mestiça sempre apresentando maior infestação. Quanto à média mensal de infestação (Figura 3), verificou-se que nas vacas mestiças a maior média foi de 125 moscas (abril/2004) e a menor de 36 moscas por animal (novembro/2004) e, na raça Guzerá, foi de 88 moscas (abril/2004) e a menor de 24 moscas por animal (agosto/2004). Esta diferença na infestação entre animais cruzados (Zebuíno x Europeu) e Zebuínos também foi encontrada por Bianchin, Koller e Detmann (2006), que verificaram que as médias de infestação dos animais descendentes do cruzamento de Bos taurus com Bos indicus eram maiores do que dos animais de sangue Nelore. Outros autores também detectaram a preferência da mosca por animais mestiços e europeus (STEELMAN et al., 1991; FARIA, 1998).

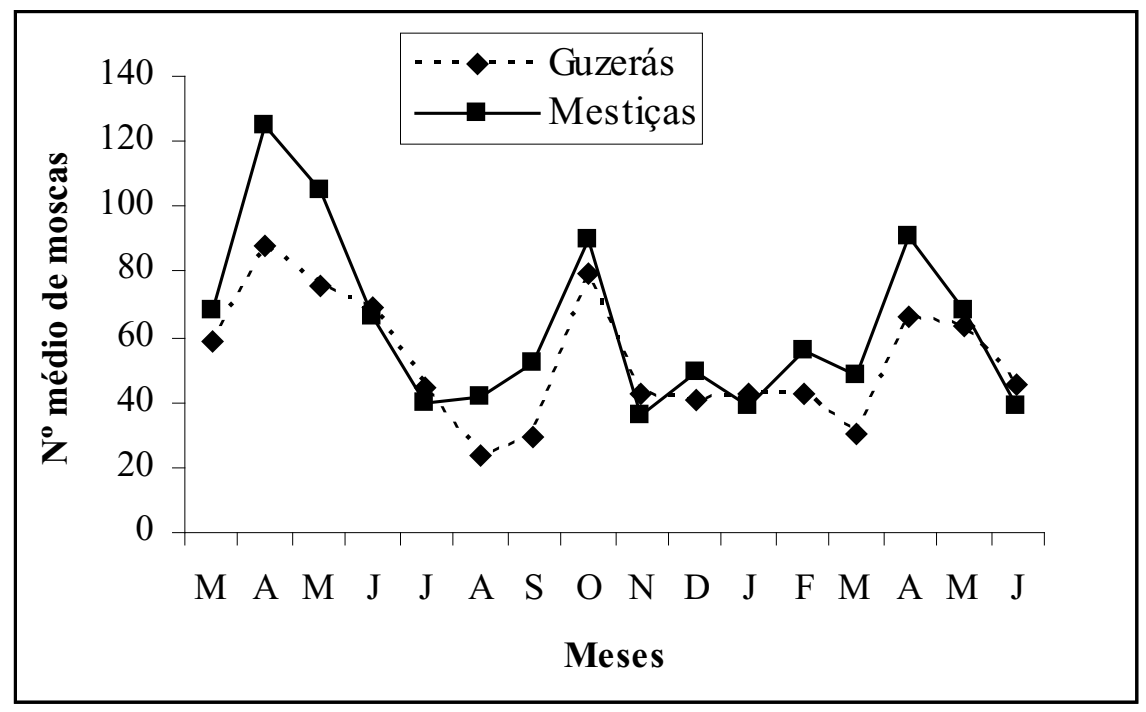

Figura 3. Número médio mensal de mosca-dos-chifres em bovinos da raça Guzerá e Mestiço ( $\mathrm{n}=15)$, no período de março de 2004 a junho de 2005, no município de Selvíria, MS.

\section{Conclusão}

Os resultados obtidos permitiram verificar que a mosca-dos-chifres está presente durante todo o ano no município de Selvíria, MS, apresentando dois picos de infestação durante o ano, um no mês de abril e outro no mês de outubro, tanto nas vacas mestiças como nas da raça Guzerá, sendo as vacas mestiças as mais infestadas.

\section{Referências}

ALMEIDA, F. A.; ZOCOLLER-SENO, M. C.; BASSO, F. C.; FILHO VALÈRIO, W. V. Comparação de métodos de contagem da mosca-dos-chifres (Haematobia irritans) em diferentes regiões do corpo de bovinos da raça
Guzerá e mestiço. In: CONGRESSO DE INICIAÇÃO CIENTÍFICA DA UNESP, 17., 2005, Ilha Solteira. Anais... Ilha Solteira, 2005. p.1-2. (CD ROM).

BARROS, A. T. M. Dynamics of Haematobia irritans irritans (Díptera: Muscidae) infestation on Nelore Catlle in the Pantanal, Brazil. Memórias do Instituto Oswaldo Cruz, Rio de Janeiro, v. 96, n. 4, p. 445-450, 2001.

BARROS, A. T. M. Situação da resistência da Haematobia irritans no Brasil. Revista Brasileira de Parasitologia Veterinária, São Paulo, v. 13, supl. 1, p. 109-110, 2004.

BIANCHIN, I.; ALVES, R. G. O. Mosca-dos-chifres, Haematobia irritans: comportamento e danos em vacas e bezerros Nelore antes da desmama. Pesquisa Veterinária Brasileira, Rio de Janeiro, v. 22, n. 3, p. 109-113, 2002.

BIANCHIN, I.; KOLLER, W. W.; ALVES, R. G. O. Efeito da mosca-dos-chifres, Haematobia irritans (L.) (Diptera: 
Muscidae), no ganho de peso de bovinos Nelore. Ciência Rural, Santa Maria, v. 34, n. 3, p. 885-890, 2004.

BIANCHIN, I.; KOLLER, W. W.; DETMANN, E. Sazonalidade de Haematobia irritans no Brasil Central. Pesquisa Veterinária Brasileira, Rio de Janeiro, v. 26, n. 2, p. 79-86, 2006.

BORDIN, E. L. A Haematobia irritans: controle químico com ivermectin formulação pour-on. A Hora Veterinária, Porto Alegre, ano. 11, n. 65, p. 20-21, 1992.

CASTRO, E.; GIL, A.; PIAGgIO, J.; CHIFFLET, L.; FARIAS, N. A.; SOLARI, M. A.; MOON, R. D. Population dynamics of horn fly, Haematobia irritans irritans (L.) (Diptera: Muscidae), on Hereford cattle in Uruguay. Veterinary Parasitology, Amsterdam, v. 151, n. 2/4, p. 286-299, 2008.

CORDOVÉS, C. O. Epizootiologia e eficácia da cipermetrina $5 \%$, formulação pour-on, em infestações naturais de Haematobia irritans. A Hora Veterinária, Porto Alegre, v. 18, n. 105, p. 77-81, 1998.

FARIA, M. J. Mosca-dos-chifres. Rio de Janeiro: PESAGRO-RIO, 1998. (Informe Técnico, n. 26).

FRANKS, R. E.; BURNS, E. C.; ENGLAND, N. C. Color preference of the horn fly, Haematobia irritans, on beef cattle. Journal Economic Entomology, Cambridge, v. 57, n. 3, p. 371-372, 1964.

GARCIA, C. A.; SALAS, S. C.; OSTI, J. L.; VÁZQUEZ, Z. G. Dinâmica poblacional de Haematobia irritans em um hato de bovinos de Soto la Marina, Taumalipas, México. Veterinário México, v. 32, n. 2, p. 149-152, abr./ jun. 2001.

GOMES, A.; KOLLER, W. W.; SILVA, R. L. Ocorrência da Haematobia irritans (Díptera: Muscidae) como vetor de Dermatobia hominis (Diptera: Cuterebridae) em Campo Grande, MS. Revista Brasileira de Parasitologia Veterinária, São Paulo, v. 7, n. 1, p. 69-70, 1998.

GRISI, L.; MASSARD, C. L.; MOYA BORJA, G. E.; PEREIRA, J. B. Impacto econômico das principais parasitoses em bovinos no Brasil. A Hora Veterinária, Porto Alegre, v. 125, p. 8-10, 2002.

GUGLIELMONE, A. A.; GIMENO, E.; IDIART, J.; FISHER, W. F.; VOLPOGNI, M. M.; QUAINO, O.; ANZIANI, O. S.; FLORES, S. G.; WARNKE, O. Skin lesions and cattle hide damage from Haematobia irritans infestations in cattle. Medical and Veterinary Entomology, v. 13, n. 3, p. 323-328, 1999.

LIMA, L. G. L.; PERRI, S. H. V.; PRADO, A. P. Variation in population density or horn flies (Haematobia irritans irritans) (L.) (Diptera: Muscidae) in Nelore catlle (Bos indicus). Veterinary Parasitology, Amsterdam, v. 117, n. 4, p. 309-314, 2003.

MARTINS, J. R.; VOLPOGNI, M. M.; CASTELLI, M.E.; GUGLIELMONE, A. A. Ação da doramectina injetável sobre Haematobia irritans em bovinos naturalmente infestados: resultados de observações simultâneas no Brasil e Argentina. Ciência Rural, Santa Maria, v. 32, n. 4, p. 633-636, jul./ago. 2002.

MARTINS, J. R.; PORCIÚNCULA, J. A.; VIEIRA, M. I. B. Dinâmica populacional da mosca-dos-chifres, Haematobia irritans (Díptera: Muscidae), em São Gabriel, região Centro-Oeste do Rio Grande do Sul. Revista Brasileira de Parasitologia Veterinária, São Paulo, v. 11, n. 2, p. 99-101, 2002.

MORGAN, N. O. Autecology of the adult horn fly, Haematobia irritans (L.) (Diptera: Muscidae). Ecology, Washington, v. 45, n. 4, p. 728-736, 1964.

PRUETT, J. H.; STEELMAN, C. D.; MILLER, J. A.; POUND, J. M.; GEORGE, J. E. Distribution of horn flies on individual cows as a percentage of the total horn fly population. Veterinary Parasitology, Amsterdam, v. 116, n. 3, p. 251-258, 2003.

SAUERESSIG, T. M. Mosca-dos-chifres, Haematobia irritans: diagnóstico e controle. Planaltina: EMBRAPA, CNPGC, 1992. (EMBRAPA CAPC. Documentos, 43).

STEELMAN, C. D.; BROWN, A. H.; GBUR, E. E.; TOLLEY, G. Interactive response of the horn fly (Diptera: Muscidae) and selected breeds of beef cattle. Journal of Economic Entomology, Cambridge, v. 84, n. 4, p. 12751282, 1991.

STEELMAN, C. D.; BROWN, M. A.; GBUR, E. E.; TOLLEY, G. The effects of hair density of beef cattle on Haematobia irritans horn fly populations. Medical and Veterinary Entomology, Oxford, v. 11, n. 3, p. 257-264, 1997.

VALÉRIO, J. R.; GUIMARÃES, J. R. Sobre a ocorrência de uma nova praga, Haematobia irritans (L.) (Diptera, Muscidae) no Brasil. Revista Brasileira de Zoologia, São Paulo, v. 1, n. 4, p. 417-418, 1983. 